\title{
Electron properties of an emissive cathode: analysis with incoherent Thomson scattering, fluid simulations and Langmuir probe measurements
}

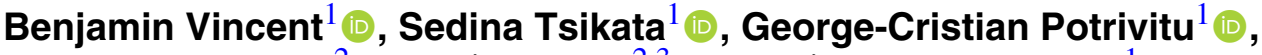 \\ Laurent Garrigues $^{2}$ (D) , Gaétan Sary ${ }^{2,3}$ and Stéphane Mazouffre ${ }^{1}$ (i) \\ ${ }^{1}$ ICARE, CNRS (UPR 3021), 1 C ave. de la Recherche Scientifique, 45071 Orléans, France \\ ${ }^{2}$ LAPLACE, Université de Toulouse, CNRS (UMR 5213), F-31062 Toulouse, France \\ ${ }^{3}$ Current address: CEA, DAM, DIF, F-91297 Arpajon, France \\ E-mail: benjamin.vincent@cnrs-orleans.fr and sedina.tsikata@cnrs-orleans.fr
}

Received 6 May 2020

Accepted for publication 4 June 2020

Published $\mathrm{xx} \mathrm{xx} \mathrm{xxxx}$

\begin{abstract}
Emissive cathodes serve a key function as the electron source for Hall plasma thrusters. Development of reliable numerical models for their operation and information on plasma behavior requires the use of sensitive and accurate diagnostics. This paper discusses results from a combined experimental and numerical study of the plasma regimes and properties of a 5 $\mathrm{A} \mathrm{LaB}_{6}$ pellet emitter cathode. Electron properties across a range of currents (2-16 A) are determined for the first time using incoherent Thomson scattering. A 2D axisymmetric fluid code is adapted to the study of the electron properties of this cathode and the nature of regime transitions. Similar trends in the numerical and experimental results are found, such as transitions to plume-like behavior with increasing current; quantitative agreement would require the use of a combined thermal-plasma model for the cathode under study. Comparisons of results from non-invasive Thomson scattering to Langmuir probe measurements reveal limitations of the latter diagnostic for plasma characterization.
\end{abstract}

Keywords: hollow cathode, numerical modeling, incoherent thomson scattering, langmuir probe, thermionic emission, fluid simulation, plasma diagnostics

(Some figures may appear in colour only in the online journal)

\section{Introduction}

Hall plasma thrusters, widely used for in-space propulsion of telecommunications satellites, require the use of external emissive cathodes. These act as electron sources for the ionization of the neutral gas propellant and for downstream neutralization of the resulting ion beam. Research into the operation modes and design optimization of emissive cathodes for thrusters has been ongoing for the past several decades [1-3]. Recent work concerns not only cathodes in the high-current (100 A) range, destined for thruster powers of several tens of $\mathrm{kW}[4,5]$, but also low-power designs [6-8] for miniature thrusters, suitable for micro- and nanosatellite applications.
In recent years, a major research effort in simulations, experiments and theory has been devoted to the study of the crossed-field discharge of the main Hall thruster plume. This has allowed the analysis of features such as plasma waves [9] which potentially affect thruster operation. Technological advances, such as the development of magnetically-shielded thruster architectures [10], offer the opportunity of extending thruster lifetimes significantly.

Increased thruster development efforts have contributed to further research into cathodes, known to be the lifetimelimiting factor for Hall thrusters. Emissive cathodes are subject to bombardment and erosion by accelerated ions [11], a phenomenon potentially linked to the development of 
ion-acoustic turbulence in the cathode plume [11-13]. Coupling between the emissive cathode and the main thruster plasma plumes [14] may affect global features such as discharge oscillations. Understanding such complex cathode physics and its influence on thruster operation requires reliable diagnostics for particle properties and the development of advanced cathode codes.

Langmuir probes $[15,16]$ are conventional diagnostics which have long been used for characterization of different cathode plasmas, such as in references [17-19]. The shape of the current-voltage characteristic obtained from an electrode inserted in the plasma is used to extract electron density and temperature, floating potential, and plasma potential. The simplicity of this diagnostic is associated with drawbacks, such as discharge perturbation [20] and restricted spatial resolution (dependent on the probe dimensions) and temporal resolution.

Incoherent Thomson scattering (ITS) is less-commonly used for electron property characterization in low-density plasmas. This diagnostic technique uses the intensity and spectral shape of the light elastically scattered by the free electrons of the plasma. The main challenges of the diagnostic are its complexity (relative to probes) and the low scattered intensity available at low plasma densities, but it has the advantage of avoiding plasma perturbation, if certain criteria are respected [21, 22] and high spatial and temporal resolution are attainable.

In addition to experimental tools, numerical simulations are the source of insights into cathode physics. Early analytical studies [23-25] on aspects of hollow cathode physics such as electron emission gave rise to increasingly sophisticated numerical models, and in particular, the first comprehensive 2D axisymmetric code OrCa2D [26], which has been applied in recent years to the study of several features, including cathode erosion and the development of oscillations and spotplume regime transitions [27-29]. Over the last few years, another 2D axisymmetric fluid code [30, 31] was developed and evaluated for the simulation of thermal and plasma physics of the NSTAR NASA hollow cathode. This code proved capable of accurately reproducing profiles for the interior and exterior electron properties measured using probes.

The availability of a sensitive, non-invasive and reliable ITS diagnostic offers the opportunity to evaluate the performance of numerical codes and determinine their ability to reproduce typical cathode operation and electron property profiles.

With this in mind, in this work, we adapt the Sary and Garrigues fluid code $[30,31]$ to studies of a 5 A MIREA cathode equipped with $\mathrm{a} \mathrm{LaB}_{6}$ pellet emitter, and investigate a range of operating regimes. The electron properties from these regimes are compared directly with ITS electron property measurements on the same cathode. This, to our knowledge, is the first work combining ITS and numerical simulation studies for emissive cathodes. A limited comparison of results to Langmuir probe measurements is also discussed.

This paper is structured as follows. The emissive cathode source and diagnostics (ITS, Langmuir probe) used for the investigations are desribed in section 2. Section 3 is devoted to the presentation of the main features of the $2 \mathrm{D}$ fluid model. In section 4, diagnostic and simulation results for electron properties, obtained under various conditions, are presented and discussed. A summary of the main insights gained is presented in section 5 .

\section{Experimental setup}

\subsection{Cathode details and operation}

The cathode was tested under vacuum in a $1.8 \mathrm{~m}$ long, $0.8 \mathrm{~m}$ diameter stainless steel vacuum vessel. A $1101 \mathrm{~s}^{-1}$ dry pump combined with a $3501 \mathrm{~s}^{-1}$ turbomolecular pump and a cryogenic pump cooled down to approximately $30 \mathrm{~K}$ gave a base pressure of $10^{-5} \mathrm{~Pa}$ and an operating pressure below at least $4 \times 10^{-3} \mathrm{~Pa}$ for all the cathode discharge operating conditions.

The cathode considered in this work is based on the model developed by the MIREA Institute and features a thermoemissive $\mathrm{LaB}_{6}$ pellet heated by a tungsten filament to supply electrons to the discharge. The cathode internal body, made of molybdenum, minimizes radiative losses dissipated by the tungsten filament. In addition, the high electrical conductivity of the internal body and $\mathrm{LaB}_{6}$ pellet guarantees electrical closure of the heating circuit connections shown in red in figure 1. The pellet is a disc $8 \mathrm{~mm}$ in diameter and $2 \mathrm{~mm}$ thick, however, the emissive surface is reduced to $6 \mathrm{~mm}$ in diameter due to the internal body architecture.

Both the experimental campaign and simulation focus on the behavior of the cathode operation in diode mode, operated independently of a thruster and using an externally-mounted anode. For the experimental campaign, the cathode was operated in self-heating mode. The experimental setup uses a stainless steel disc anode to establish the potential drop through which the electrons are accelerated, of diameter $110 \mathrm{~mm}$ and with a central hole of diameter $21 \mathrm{~mm}$. This anode directly faces the $3 \mathrm{~mm}$-diameter cathode orifice and is located at an axial distance of $40 \mathrm{~mm}$. Figure 1 shows the basic architecture of this cathode and anode mounting. The cathode is operated over a range of discharge currents (2-16 A) and xenon flow rates $\left(0.2-0.8 \mathrm{mg} \mathrm{s}^{-1}\right)$.

Two operating modes named the 'spot' and 'plume' modes are commonly identified for cathode discharge operation in the diode configuration [1]. The spot mode is u sually the operation mode desired and is associated with high electron density, low electron temperature (Csiky identified this mode with temperatures in the range of $0.5 \mathrm{eV}$ [2]) and low discharge oscillation. The plume mode is associated with lower electron density, higher electron temperature (in the range of 1 $2 \mathrm{eV}$ in reference [2]) and higher oscillations of the discharge voltage and current (a ratio $I_{d, s t d} / I_{d \text {, mean }}$ above few percent is usually attributed to the plume mode [32]), leading to a lower cathode lifetime [33]. The experimental work presented in this article focuses on discharge conditions for which the cathode discharge is in the so-called spot mode.

\subsection{Incoherent Thomson Scattering diagnostic}

A complete description of the design of the incoherent Thomson scattering diagnostic, as well as the procedures used for 


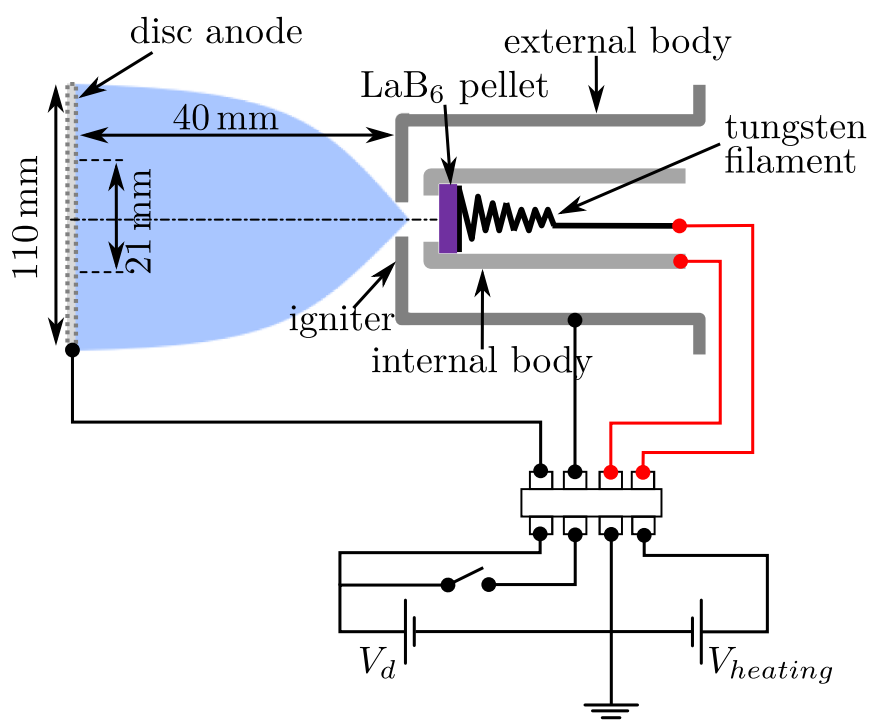

Figure 1. Diagram of the MIREA cathode architecture and electrical connections. The cathode is operated in diode mode with an external disc anode. The plasma region is shown in blue.

signal detection and calibration, can be found in detail in reference [34]. This section makes a summary of the main features of the diagnostic and the specificities related to its implementation on the cathode.

\subsubsection{Summary of diagnostic design. Electron property} measurements were performed using the recently-developed incoherent Thomson scattering diagnostic named THETIS [34]. A key feature of this diagnostic is the use of a recently-developed volume Bragg grating (VBG) notch filter to efficiently limit stray light transmission near the incident laser wavelength, while preserving a high transmission efficiency outside the band-stop region. This diagnostic design allows a simpler detection branch in comparison to diagnostics using triple grating spectrometers and significantly reduces transmission losses, resulting in a high degree of sensitivity.

The diagnostic setup is shown in figure 2. It is comprised of (i) a transmission branch, used to produce the Thomson scattering signal (this branch is shown in dark green in figure 2), and (ii) a detection branch, used to collect the scattered light over a reduced solid angle, filter the sources of stray light, spectrally disperse the photons collected, and measure the scattered signal with an intensified CCD camera (this branch is shown in light green in figure 2).

The light source of the transmission branch is a 10 $\mathrm{Hz}$ pulsed, frequency-doubled Q-switch Nd:YAG laser (wavelength of $532 \mathrm{~nm}$, pulse energy of $430 \mathrm{~mJ}$ and pulse width of $5 \mathrm{~ns}$ ). The initial vertically polarized $9 \mathrm{~mm}$ diameter beam is expanded with a magnification of 3 and focused with a $2 \mathrm{~m}$-focal length lens in the plasma volume. To reduce stray-light propagation the beam passes through two Brewster windows mounted at the end of tubes inside which apertures are placed. The beam ends its path in a large-aperture beam dump.
Scattered light is collected by the detection branch and imaged onto the entrance of a $5 \times 3$ matrix of fibers $(0.3 \mathrm{~mm}$ in individual diameter and $\mathrm{NA}=0.22$ ). Light is focused onto the fibers with a $200 \mathrm{~mm}$ focal length, $100 \mathrm{~mm}$ diameter biconvex lens positioned at $520 \mathrm{~mm}$. The fiber bundle conducts the light to the optical bench where it is collimated and filtered by the VBG notch filter. The filtered light is then transmitted to the spectrometer through a $0.3 \mathrm{~mm}$ wide slit and dispersed with a 2400 lines $\mathrm{mm}^{-1}$ holographic grating. An intensified camera, triggered on the laser Q-switch signal, measures the spectrum of the remaining photons over a $15 \mathrm{~ns}$ temporal window.

A translation stage allowed measurement from $1 \mathrm{~mm}$ to $10 \mathrm{~mm}$ from the orifice. Due to the collection optics magnification, the size of the fiber bundle and the size of the laser spot, the observation volume over which electron properties are averaged can be considered as a cylinder $0.3 \mathrm{~mm}$ in diameter and $2.5 \mathrm{~mm}$ in length. Given the orientation of the detection branch relative to the transmission branch, electron properties are probed in the $(y z)$ plane at an angle $45^{\circ}$ relative to the $y$ direction.

2.2.2. Procedure for data analysis. To obtain quantitative data, the detection branch of the THETIS diagnostic was calibrated with the same acquisition parameters as for Thomson scattering measurements.

The spectral calibration was performed with a low-pressure neon gas lamp. The instrument function (which characterizes the light redistribution induced by the detection branch) was estimated using the spectrum measured from the background signal generated by the laser reflections. These sources of light from the laser were considered perfectly monochromatic in comparison to the spectrometer resolution. These spectral calibrations were performed with the VBG filter removed, and used to quantitatively analyze the spectral distribution of the signals acquired.

The intensity calibration was performed using the fitting of the Raman scattering spectrum, measured from a known pressure of nitrogen, and with the energy of the laser, monitored after each acquisition. The VBG notch filter was tuned for stray light rejection at $532 \mathrm{~nm}$. With this calibration, the total intensity of the signal from a given scattering process can be used to determine the density of the scattering particles when the corresponding scattering cross-section and intensity of the incident light are well-characterized.

Examples of Thomson scattering spectra obtained from cathode experiments for two different discharge currents are shown in figure 3, with corresponding electron properties indicated in the caption. From these spectra, the electron temperature is deduced from the spectral width and the density is estimated from the total intensity scattered. These spectra show the spectral region of rejected data (corresponding to the residual stray light within the notch filter bandstop, in orange), the full set of experimental points used for the analysis procedure (in blue) and the green fit line from which Gaussian fit parameters are determined to deduce the electron properties. As is clear from figure 3(a), the high sensitivity of this 


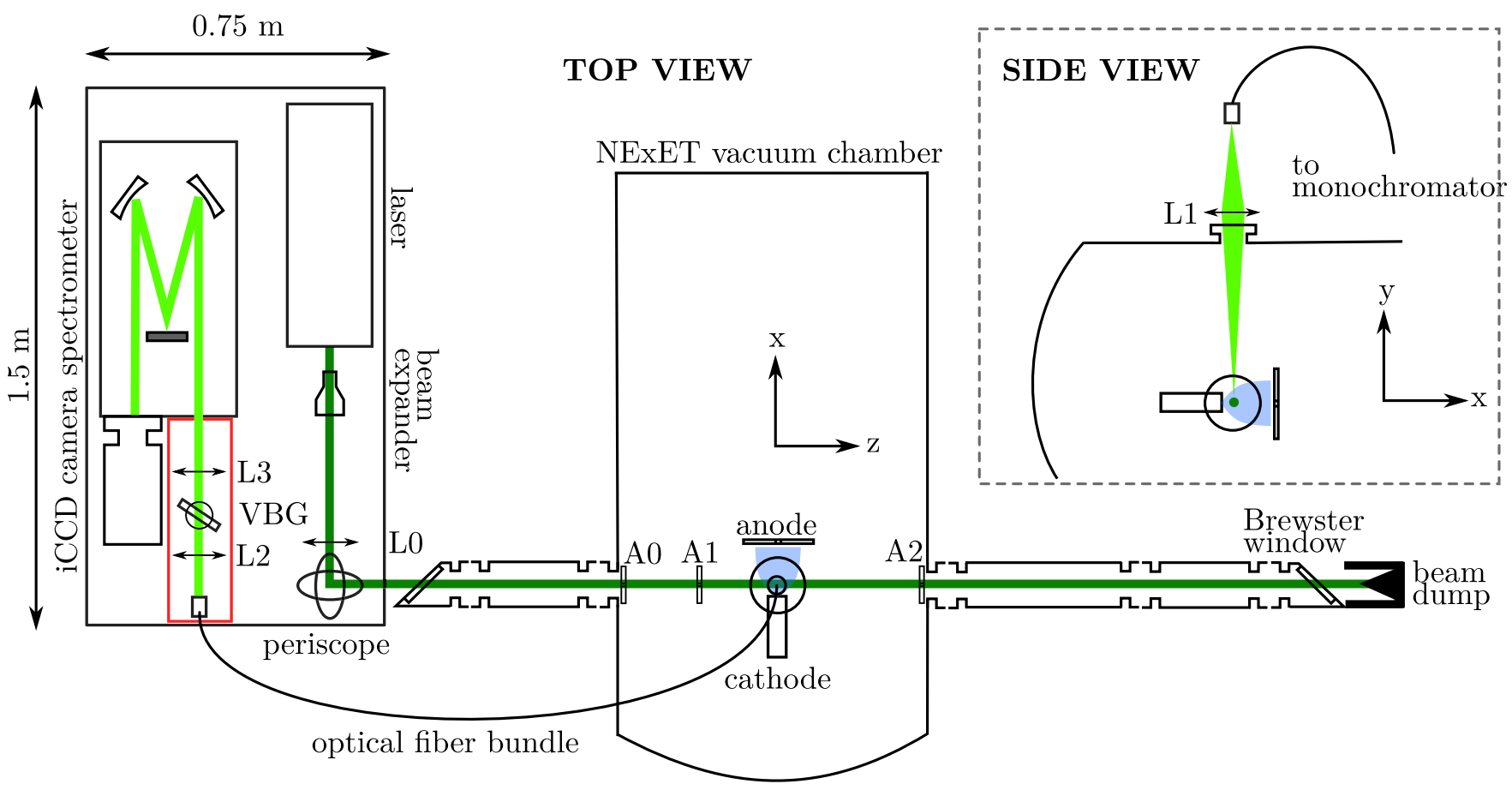

Figure 2. View of the THETIS incoherent Thomson scattering bench setup for cathode investigations (not to scale). Two views are shown: a top view of the bench and laser beam trajectory (in dark green) and a side view, showing the collection of scattered light (in light green) at the top of the vacuum vessel. L0, L1, L2, and L3 indicate lenses; A0, A1, and A2 indicate apertures; VBG indicates the volume Bragg grating notch filter used for stray light suppression.

diagnostic allows detection of Thomson signal amplitudes of only a few counts, which corresponds to electron densities in the $10^{16} \mathrm{~m}^{-3}$ range.

\subsection{Langmuir probe}

Probe measurements were performed with a single cylindrical Langmuir probe made with a $0.10 \mathrm{~mm}$-diameter tungsten wire, protruding $1 \mathrm{~mm}$ from a single-bore alumina tube. Measurements were performed on the cathode axis, with the probe oriented along the $\mathrm{x}$-direction (cf figure 2). In addition to the three-axis displacement stages of the cathode, the probe was placed on a translation plate for quick displacement along the $x$-axis. The resolution of electron properties along the $x$-axis with the Langmuir probe is assumed to be approximately the size of the probe itself, with the influence of the sheath and imprecision of translation stages neglected. The acquisition of the $I-V$ characteristics was achieved with an Impedans ALP system. A 1:11 attenuator was used when the collected current level exceeded that of the analog-to-digital converter dynamics.

Because the plasma outside the cathode orifice is collisionless (electron mean free path much greater than the probe dimensions) and unmagnetized, the Druyvesteyn method [35] was used to extract information on electron properties from the $I-V$ characteristic. To do so, the $I(V)$ characteristic was first filtered with a fourth order Butterworth filter and then smoothed using a Savitzky-Golay filter [36]. The plasma potential was obtained from the root of the second derivative of the $I(V)$ curve (calculated by the Savitzky-Golay filter). The
Druyvesteyn formula was applied to this second derivative to obtain an EEDF (not normalized):

$$
F(\epsilon)=\frac{4}{e^{2} A_{p}}\left(\frac{m \epsilon}{2 e^{2}}\right)^{1 / 2} \frac{d^{2} I}{d V^{2}}\left(J^{-1} m^{-3}\right)
$$

where $A_{p}$ is the probe collection area, $\varepsilon=e\left(V_{p}-V\right)$ the electron energy and $V_{p}$ the plasma potential estimated with the position of the maximum of the first derivative of the $I(V)$ function.

The normalization coefficient of equation (1) within the interval boundary starting from zero electron energy (when the probe potential is at plasma potential) up to the maximum electron energy (in principle, obtained at the minimum probe potential scanned) give the electron density:

$$
n_{e}=\int_{0}^{+\infty} F(\epsilon) d \epsilon\left(m^{-3}\right)
$$

Using the hypothesis of a Maxwell-Boltzmann Electron Velocity Distribution Function (EVDF) and isotropic electron behavior, the normalized $\operatorname{EEDF} f(\varepsilon)=F(\varepsilon) / n_{e}$ is a chi-squared distribution with three degrees of freedom. The calculation of the first moment of this distribution gives:

$$
\mathbb{E}[\epsilon]=\int_{0}^{+\infty} \epsilon f(\epsilon) d \epsilon=\frac{3}{2} k_{B} T_{e}(J)
$$

Under the hypothesis of a chi-squared experimental EEDF we obtain :

$$
T_{e}=\frac{2}{3 k_{B}} \mathbb{E}[\epsilon](K)
$$




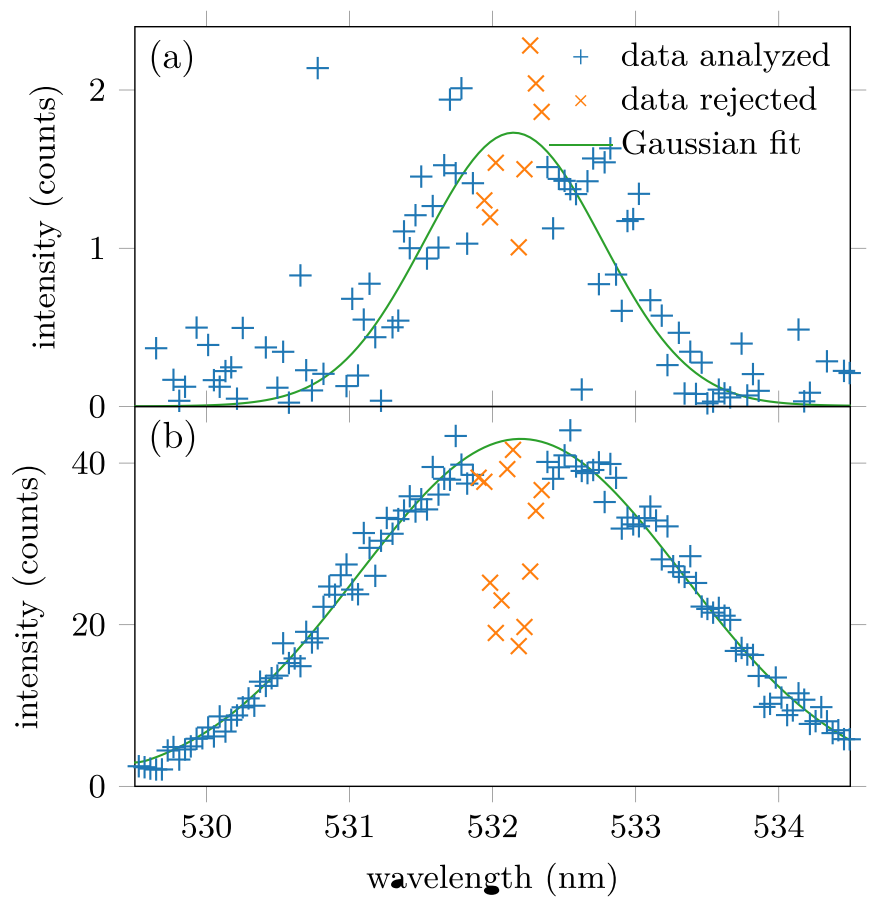

Figure 3. Examples of Thomson spectra obtained on the cathode, illustrating the diagnostic sensitivity. These examples correspond to (a) 2 A discharge current, $6 \mathrm{~mm}$ from the cathode orifice, $T_{e}=$ $0.50(5) \mathrm{eV} ; n_{e}=2.0(2) \times 10^{16} \mathrm{~m}^{-3}$ and (b) 16 A discharge current, $1.3 \mathrm{~mm}$ from the cathode orifice, $T_{e}=1.2(1) \mathrm{eV} ; n_{e}=9.5(8) \times 10^{17}$ $\mathrm{m}^{-3}$. Experimental points, Gaussian fit line and rejected points are shown in blue, green and orange, respectively.

\subsection{Uncertainty analysis}

Systematic and random uncertainties may be considered relevant to this work. For the uncertainty analysis of the experiments presented, only systematic uncertainties were taken into account. To determine the random uncertainty, measurements would have to be repeated many times. For example, due to fast probe aging in the plasma studied, a new probe for each Langmuir measurement would have to be used; this would not have been feasible within the restricted time available. For the Thomson measurements, each spectrum requires 20 min of acquisition (10 min for the Thomson scattering signal and $10 \mathrm{~min}$ for the background signal accumulations for subtraction). The time constraint is again the limiting factor. The uncertainties were calculated using a propagation of error calculation involving the quadratic sum of systematic uncertainties in the parameters used to calculate $n_{e}$ and $T_{e}$.

2.4.1. Thomson scattering uncertainties. Uncertainties in $T_{e}$ and $n_{e}$ from Thomson measurements have few contributions from the experiment equipment. The main experimental contribution is from laser power root mean square (r.m.s) fluctuations. Statistics on the laser energy measured over $10 \mathrm{~min}$ during background acquisitions confirmed an r.m.s variation below 1\%. The use of a high precision Inficon CDG025D capacitive diaphragm pressure gauge enabled a relative precision of $0.2 \%$ in the pressure measurement. The two main sources of uncertainties are from the data analysis phase. For data analysis of the calibration spectrum, a Raman cross-section with an uncertainty of $8 \%$ is used [37]. In addition, each spectrum fitting is obtained with an uncertainty whose value is linked to the signal-to-noise ratio level; to take this contribution into account, the covariance matrix was extracted for each spectrum fit.

2.4.2. Langmuir probe uncertainties. For probes, accounting for errors associated with the EEDF method used to determine electron properties is a rather complex task. Herman and Gallimore [38] predict an error of $\pm 8 \%$ in the determination of the EEDF using the Druyvesteyn method, but only when all the constitutive parameters that enter into the EEDF computation are known to a high degree of accuracy. The plasma potential magnitude remains the main source of uncertainty in computing the EEDF. The characteristic times and filtering circuits of the Impedans ALP System, together with data processing (filtering, numerical differentiation) induce systematic uncertainties in the plasma property estimations. If the first derivative of the probecollected current is used for determining the plasma potential, the accuracy of the potential measurement is approximated to $\pm 10 \%$ [39]. The following results use an uncertainty in plasma density of $\pm 50 \%$ and $\pm 30 \%$ for the electron temperature [40].

\section{Numerical model}

As mentioned in the introduction, this work seeks to compare numerical results on electron properties for the $5 \mathrm{~A}$ MIREA cathode with properties measured directly in experiments. A particular focus is placed on ITS as a new diagnostic. This work follows (i) application in 2017 of our code $[30,31]$ to a different standard cathode, and (ii) recent validation of the performance of the ITS diagnostic [34]. This research seeks to build on the existing body of experiment-versus-simulation comparisons, many of which have been performed in studies using the well-established OrCa2D [26] code. Full details on the numerical method are presented in reference [30], and are only briefly summarized here.

\subsection{Governing equations}

The numerical scheme considers three species: Xe atoms, $\mathrm{Xe}^{+}$ions, and electrons, with the multiply-charged ion population are neglected. Maxwellian velocity distributions are considered to apply for all species in this plasma. Inside the cathode, the transport of species is dominated by collisions between electrons and neutrals $(e-n)$, between 
ions and neutrals $(i-n)$, and Coulomb collisions between electrons and ions $(e-i)$. The plasma is assumed to be quasineutral with the ion density $n_{i}$ equal to the electron density $n_{e}$.

The mass conservation equation for the ions can be written as:

$$
\frac{\partial n}{\partial t}+\nabla \cdot\left(n \vec{u}_{i}\right)=S
$$

where $\vec{u}_{i}$ is the ion velocity.

The mass conservation equation for the neutrals can be written as:

$$
\frac{\partial n_{n}}{\partial t}+\nabla \cdot\left(n_{n} \vec{u}_{i}\right)=-S
$$

where $n_{n}$ is the neutral density. In equations (5) and 6, $S=$ $n n_{n} k_{i o}\left(T_{e}\right)$ is the source term and $k_{i o}$ the ionization rate coefficient.

The momentum conservation equations for $\mathrm{Xe}^{+}$ions, neglecting viscosity and magnetic field, are written:

$$
M\left(\frac{\partial}{\partial t}\left(n \vec{u}_{i}\right)+\nabla \cdot\left(n \vec{u}_{i} \otimes \vec{u}_{i}\right)\right)=-n e \nabla \phi-\nabla p_{i}+M S \vec{u}_{n}
$$

where $\phi$ is the plasma potential.

The following electron drift diffusion equation is used:

$$
\vec{j}_{e}=-e n \mu_{e} \nabla \phi+\mu_{e} \nabla p_{e}
$$

with the electron pressure $p_{e}=n k_{B} T_{e}$ and the electron mobility $\mu_{e}=e / m \nu_{e}$, and charge conservation is written as:

$$
\nabla \cdot\left(\overrightarrow{j_{i}}+\overrightarrow{j_{e}}\right)=0
$$

giving the expression for the plasma potential

$$
\nabla \cdot\left(e n \mu_{e} \nabla \phi\right)=\nabla \cdot\left(\mu_{e} \nabla p_{e}+e n \vec{u}_{i}\right)
$$

Lastly, energy conservation for the different species is written as follows:

for neutrals:

$$
\begin{array}{r}
\frac{\partial}{\partial t}\left(\frac{3}{2} p_{n}\right)+\nabla \cdot\left(\frac{5}{2} p_{n} \vec{u}_{n}+\vec{q}_{n}\right) \\
=\vec{u}_{n} \cdot \nabla p_{n}-\frac{3}{2} k_{B} T_{n} S-\vec{u}_{s} \cdot \nabla \cdot \vec{T}+\nabla \cdot\left(\overrightarrow{\bar{T}} \vec{u}_{s}\right) \\
+Q_{n}-\left(\vec{R}_{i s o, i \rightarrow n}+\vec{R}_{e \rightarrow n}\right) \cdot \vec{u}_{n}
\end{array}
$$

where the neutral thermal conduction flux is $\vec{q}_{n}=-\lambda_{n} \nabla T_{n}$, $Q_{n}=n \bar{\nu}_{i s o} k_{B}\left(T_{i}-T_{n}\right)+3 \frac{m}{M} n n_{n} k_{e, n}^{m} k_{B}\left(T_{e}-T_{n}\right)$ and $\vec{R}_{s \rightarrow t}$ is the momentum exchange due to elastic collisions between species $s$ and $t$.

for ions:

$$
\begin{array}{r}
\frac{\partial}{\partial t}\left(\frac{3}{2} p_{i}\right)+\nabla \cdot\left(\frac{5}{2} p_{i} \vec{u}_{i}+\vec{q}_{i}\right) \\
=\vec{u}_{i} \cdot \nabla p_{i}+\frac{3}{2} k_{B} T_{n} S+\frac{1}{2} M S\left(\vec{u}_{i}-\vec{u}_{n}\right)^{2}
\end{array}
$$

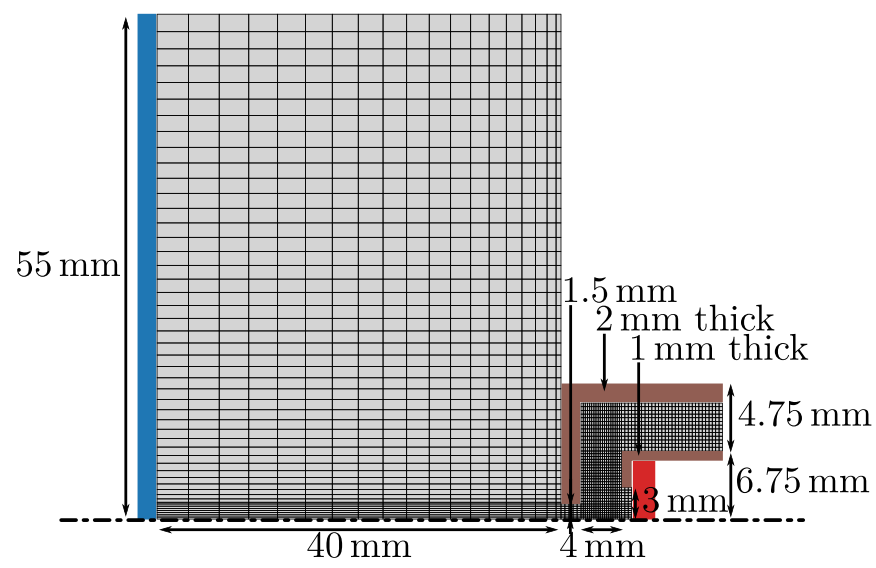

Figure 4. Simulation region analyzed with the fluid code to match MIREA architecture. The disc anode is shown in blue, and the disc $\mathrm{LaB}_{6}$ pellet emitter in red. Note that the domain is cylindrical.

$$
+Q_{i}-\left(\vec{R}_{i s o, n \rightarrow i}+\vec{R}_{e \rightarrow i}\right) \cdot \vec{u}_{n}
$$

where $Q_{i}=n \nu_{i n} k_{B}\left(T_{n}-T_{i}\right)+3 \frac{m}{M} n \nu_{i e} k_{B}\left(T_{e}-T_{i}\right)$ and $\overrightarrow{q_{i}}=$ $-\lambda_{i} \nabla T_{i}$

for electrons:

$$
\begin{aligned}
& \frac{\partial}{\partial t}\left(\frac{3}{2} p_{e}\right)+\nabla \cdot\left(\frac{5}{2} p_{e} \vec{u}_{e}+\vec{q}_{e}\right) \\
= & \vec{u}_{e} \cdot \nabla p_{e}+Q_{e}+m \nu_{e} n \vec{u}_{e}^{2}-S \epsilon_{i o}
\end{aligned}
$$

where $Q_{e}=3 \frac{m}{M} n \nu_{i e} k_{B}\left(T_{i}-T_{e}\right)+3 \frac{m}{M} n n_{n} k_{e, n}^{m} k_{B}\left(T_{n}-T_{e}\right), \vec{q}_{e}=$ $-\lambda_{e} \nabla T_{e}$ and $\varepsilon_{i o}$ is the energy lost per ionization, i.e. $12.1 \mathrm{eV}$, if only the first ionization energy of xenon is considered.

\subsection{Simulation domain and boundary conditions}

The existing simulation domain is adapted to match that of the MIREA cathode under study. Figure 4 shows the mesh with respect to the cathode dimensions and architecture. The figure shows the cylindrical simulation domain applied to half of the axisymmetric cathode, along with the dimension and placement of the anode (blue) and $\mathrm{LaB}_{6}$ pellet emitter (red).

The plasma simulation domain is discretized using a Cartesian mesh of 1880 nodes (710 in the internal region of the cathode). Further details on the discretization method are provided in reference [30]. The mesh size is at its smallest between the emitter and the orifice with a square cell size of $0.215 \mathrm{~mm}$.

For the plasma conditions inside the cathode orifice, the Debye length, at the micrometer scale, cannot be resolved by the mesh. Instead, as discussed in detail in reference [30], the wall sheaths are modeled using boundary conditions accounting separately for (i) thermionic emission of the $\mathrm{LaB}_{6}$ pellet, and (ii) the conducting nature of the cathode body.

The calculations of ion and electron fluxes and energy losses deposited on the metallic parts of the cathode (in brown in figure 4) can be treated analytically, assuming a 
non-collisional sheath with known plasma properties at the sheath edge (plasma density, electron temperature and sheath potential drop) [41]. We assume that ions lost on the walls are recombined into neutral atoms at the temperature of the wall. A slip-length boundary condition is used for neutrals, which nullifies the neutral velocity parallel to the wall at a distance below the boundary (this distance can be estimated from the fluid viscosity). The normal component of the flux is set to the recombined ion flux.

At the gas inlet (not shown in figure 4), we assume a radial profile of the neutral velocity normal to the inlet given by a Poiseuille profile. A reference density is chosen based on the density in the closest domain cell and the velocity is then computed from the desired value of the xenon mass flow injected. The temperature of the gas corresponds to the cathode body temperature. Regarding the anode plane (in blue in figure 4), a uniform electron current density is assumed (whose integration yields the given electron current at the anode). Ions are accelerated at the Bohm velocity and recombine as atoms. The external cathode body is floating and its potential is determined through a balance of positively- and negatively-charged current that impacts its surface. A complete description of the specific treatment of the open boundary of the plume region, which is not a physical boundary, can be found in reference [30].

Figure 5 shows 2D maps of electron temperature and density obtained from the simulation of the cathode discharge with two different thermionic emitter temperatures (1900 and $2050 \mathrm{~K}$ ). As shown in reference [31], similar maps can be obtained for the neutral density, neutral temperature, ion temperature and plasma potential, for example. The maps of figure 5 correspond to snapshots of the steady state solutions obtained after a simulation run time of about $5 \mathrm{~ms}$. The color scale for density and temperature has been adapted in order to highlight variations in the electron properties outside the cathode orifice, where the laser scattering measurements were performed.

\subsection{Thermionic emission description}

Particular attention must be paid to the treatment of thermionic emission of electrons from the emitter (in red in figure 4). The electron current density emission is given by the RichardsonDushman law:

$$
J_{e}^{e m}=A_{0} T_{w}^{2} \exp \left(\frac{-e \phi_{b}}{k_{B} T_{w}}\right)
$$

where $A_{0}=119.58 \mathrm{~A} \mathrm{~cm}^{-2} \mathrm{~K}^{-2}$ is Richardson's constant, $T_{w}$ is the emitter temperature, and $\phi_{b}=\phi_{W F}-\phi_{S H}$ a potential barrier that includes $\phi_{W F}$, the temperature-dependent work function, and $\phi_{S H}$, the work function lowering due to the Schottky effect.

To calculate $\phi_{b}$, a self-consistent model has been established to describe the continuous transition from the space charge-limited to thermionic emission regimes that can occur in the sheath in front of the emitter. This model, detailed in reference [30], is an extension of the work of Lin and Eng [42].
It requires, as input plasma properties at the sheath edge, electron density $n_{e}$, electron temperatures $T_{e}$ and $T_{w}$ (of the bulk plasma and at the wall, respectively), electric potential $\phi_{0}$, and electron emission at the walls.

For the electron emission, the temperature dependence of the work function $\phi_{W F}\left(T_{w}\right)$ uses the experimental data of reference [12]:

$$
\phi_{W F}\left(T_{w}\right)=2.66+1.23 \times 10^{-4} T_{w}[e V]
$$

In practice, we calculate a priori the emitted electron current density $J_{e}^{e m}$ as a function of the emission model parameters $\left(n_{0}, \phi_{0}, T_{e}, T_{w}\right)$ for a large range of parameter variations. During the 2D simulations, the emitted electron current density is simply extrapolated from pre-tabulated values.

In this work, we studied the impact of the emitter temperature on plasma properties.

In recent numerical studies of Sary et al, two cases were considered: (i) a validation case, first using an imposed emitter temperature profile [30] (an uncoupled plasma-thermal model), based on experiments on the NSTAR cathode performed by Katz et al [43], and (ii) a coupled plasma-thermal model [31].

reference [30] introduced a new model to describe electron emission in both the space charge limited regime and thermionic emission limited regime. The model of reference [30] proved capable of reproducing electron properties for the imposed temperature profile.

In this work, a slightly different approach to the work of references $[30,31]$ is taken. Firstly, as the exact thermal design of the MIREA cathode was not available, we opted not to investigate a full coupled plasma-thermal model as yet. Secondly, for the uncoupled model, rather than imposing a temperature profile for the emitter, we imposed a single uniform temperature for this element. This simplified approach is, to a certain extent, justified when one considers the very different architectures of the NSTAR cathode (which uses an emissive cylinder which develops a natural temperature gradient along its length) and the present MIREA cathode, which uses a simple emissive pellet.

The emitter temperature is treated as a parameter indirectly characterizing the emission properties of this cathode. In the simulation, the variation of this temperature is used to scan different modes of cathode operation and electron properties. This variation is performed using a lower bound for the emitter temperature which was previously measured with a thermocouple at similar discharge conditions.

In recent cathode numerical studies, Guerrero et al [44] have used the OrCa2D plasma code of reference [26] coupled self-consistently with a thermal model derived using COMSOL. In order to evaluate the performance of our plasmathermal code, a thermal model for the MIREA should be developed in the future.

\subsection{Ion acoustic instability}

In the NASA NSTAR cathode, the experimental evidence of fluctuating potential responsible for ion backflow on the 


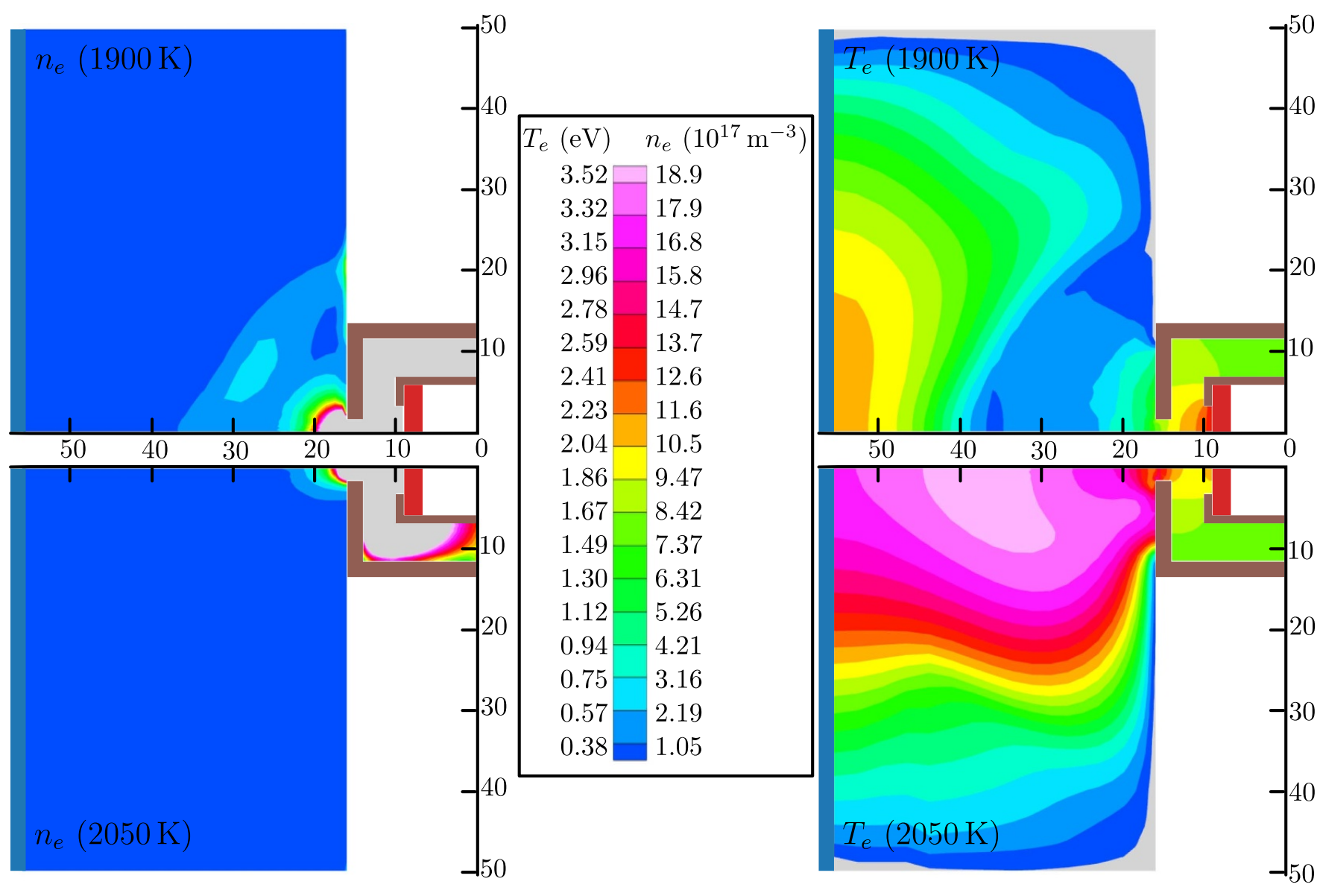

Figure 5. Time-averaged 2D maps of the electron density (left) and temperature (right) obtained from the simulation of the cathode discharge at steady state with $8 \mathrm{~A}$ of discharge current, $0.8 \mathrm{mg} \mathrm{s}^{-1}$ of xenon flow rate and for a electron emitter temperature of $1900 \mathrm{~K}$ (top) and $2050 \mathrm{~K}$ (bottom). The $x$-axis and $y$-axis coordinates are indicated in $\mathrm{mm}$. The color scale for density and temperature has been adapted in order to highlight variations in the electron properties outside the cathode orifice, where the laser scattering measurements were performed.

keeper cathode has been linked to an enhanced resistivity in the cathode plume, first proposed in reference [45]. The drop of electron density induces an increase of electron velocity to maintain the desired electron current. As a result, the relative velocity between electrons and ions can exceed the ion acoustic velocity, exciting the ion acoustic instability (IAI). Sary et al have implemented a numerical model of the acoustic instability for the cathode plume (largely inspired by the work of Davidson and Krall [46]). The IAI is modeled through an energy equation that is solved for the fluctuating electric field $\varepsilon_{f}$ :

$$
\frac{\partial \varepsilon_{f}}{\partial t}+\vec{\nabla} \cdot\left(\frac{\vec{V}_{d}}{\left|\vec{V}_{d}\right|} c_{s, i} \varepsilon_{f}\right)=2 \gamma_{M} \varepsilon_{f}
$$

where $\vec{V}_{d}=\vec{u}_{e}-\vec{u}_{i}$ is the drift velocity and $c_{s, i}=\sqrt{k B T_{e} / M}$ is the ion acoustic velocity. $\gamma_{M}$ the maximum growth rate (only the fastest-growing mode is retained and detailed in reference [30]). Assuming that the Mach number $M_{e}=\left|\vec{V}_{d}\right| / c_{s, i} \gg 1$, and $\gamma_{M} \ll \omega_{p e}, \omega_{p e}$ being the electron plasma frequency, we can then link the anomalous electron collision frequency to the electron temperature, plasma density, and fluctuating electric field $\varepsilon_{f}$ :

$$
\nu_{e_{A N}}=2 \sqrt{\pi} \omega_{p e} \frac{\varepsilon_{f}}{n k_{B} T_{e}}
$$

Thermal terms in the energy conservation equation of ions and electrons have also been included. The system of equations here described is valid only for the description of the growth rate of the IAI in the quasi-linear regime, when ion and electron energy distribution functions are almost Maxwellian. It cannot describe the saturation mechanism of the instability due to kinetic effects. Using a derivation from particle-incell simulations, Davidson and Krall have proposed to limit the maximum of $\varepsilon_{f}[46]$ :

$$
\left[\varepsilon_{f}\right]_{M A X}=\alpha_{n T_{e}} n k_{B} T_{e}, \text { where } \alpha_{n T_{e}} \in\left[10^{-3}, 10^{-2}\right]
$$

Results presented in reference [31] for a $\mathrm{BaO}$ NASA NSTAR cathode have shown that $\alpha_{n T_{e}}=3 \times 10^{-3}$ gives a reasonable agreement with experiments. We use the same value for this study.

The time-averaged values of electron temperature simulated in reference [31] were weakly affected by the saturation threshold of the acoustic ion instability. With a higher saturation threshold, the electron temperature values oscillated more strongly, but the time-averaged values remained 


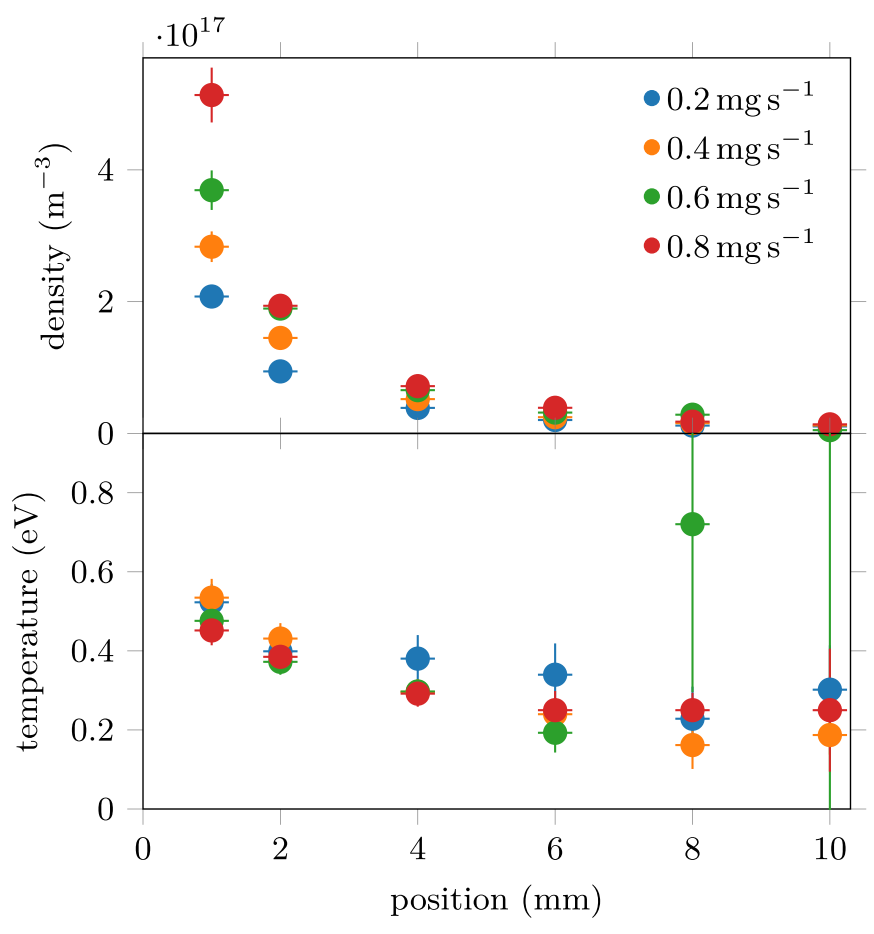

Figure 6. Electron density and temperature obtained by ITS at various distances from the cathode orifice. Measurements were performed for xenon flow rates from $0.2 \mathrm{mg} \mathrm{s}^{-1}$ to $0.8 \mathrm{mg} \mathrm{s}^{-1}$ with a fixed discharge current of $2 \mathrm{~A}$.

unchanged. The focus of this work is temporally-averaged values of electron parameters, for which we do not expect the ion-acoustic instability effect on temperature to be necessarily visible.

A brief discussion of the influence of $t$ he e mitter temperature on the appearance of discharge current oscillations compatible with transit time oscillations and the ion acoustic instability can be found in reference [47]. Qualitative agreement between experiments and calculations is also reported in reference [47] when the cathode is operated in non-self-heated mode. It is important to note that the fluid treatment of the species in this code would limit, to some degree, the ability of the model to quantitatively describe features in the plume.

\section{Results and discussion}

\subsection{Electron properties from ITS measurements}

Electron density and temperature are determined for various flow rates and discharge currents. Generally, as the dense plasma created inside the cathode expands outside over a wide solid angle, a decrease of the neutral, ion, and electron density is expected, along with the electron temperature.

4.1.1. Xenon flow rate exploration. Axial measurement of electron properties with a discharge current of $2 \mathrm{~A}$ and xenon flow rate between $0.2 \mathrm{mg} \mathrm{s}^{-1}$ and $0.8 \mathrm{mg} \mathrm{s}^{-1}$ were made using the ITS diagnostic. Results are shown in figure 6.

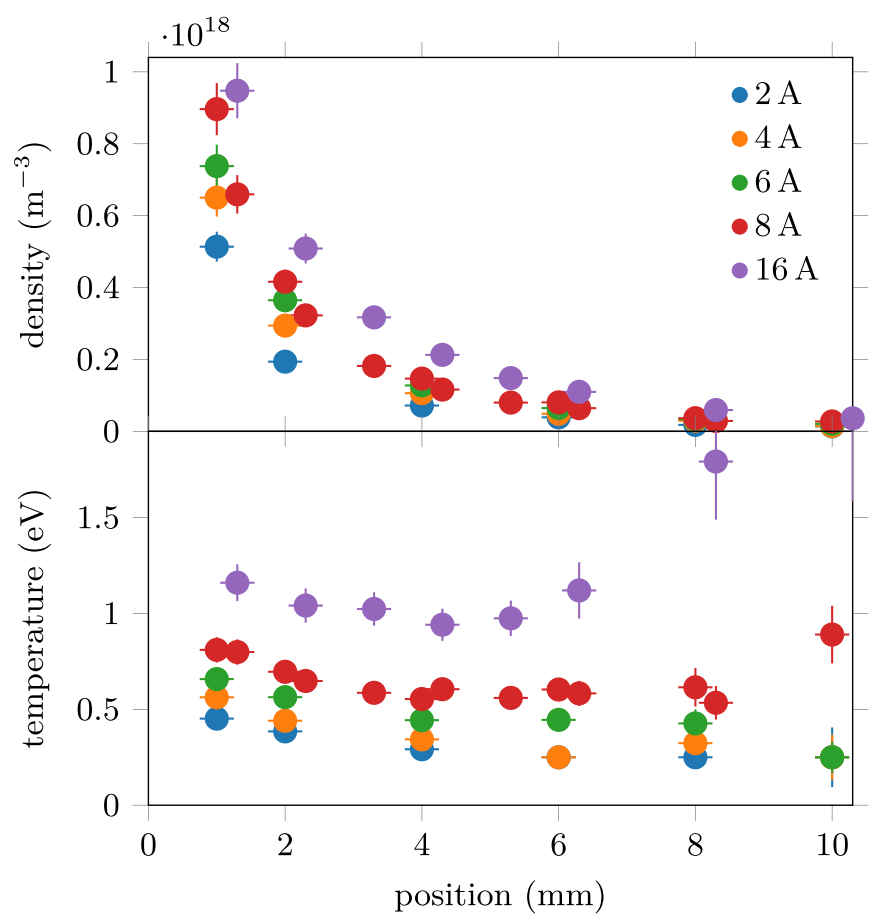

Figure 7. Electron density and temperature obtained by ITS at various distances from the cathode orifice. Measurements were performed for discharge currents from $2 \mathrm{~A}$ to $16 \mathrm{~A}$ with a fixed xenon flow rate of $0.8 \mathrm{mg} \mathrm{s}^{-1}$.

It is observed that an increase of xenon flow induces an increase of electron density. This increase is more pronounced close to the orifice and can be explained under the hypothesis of a steady ionization rate. At higher xenon flow rate, neutral density, and consequently electron density, rise if the ionization rate remains constant.

For the electron temperature, a slight decrease is observed at a higher xenon flow rate. This is due to an increased rate of electron collisions with neutrals or ions with higher xenon flow.

4.1.2. Discharge current exploration. Axial measurements were performed with a fixed xenon flow rate of $0.8 \mathrm{mg} \mathrm{s}^{-1}$ and discharge currents between $2 \mathrm{~A}$ and $16 \mathrm{~A}$. Results are shown in figure 7 .

Here, an increase in discharge current induces an increase in electron temperature. At low currents $(2 \mathrm{~A}$ to $6 \mathrm{~A})$ electron temperature decreases monotonically with distance. However, for higher currents (6 A to $16 \mathrm{~A}$ ), this trend changes: an increase of electron temperature at distances greater than $5 \mathrm{~mm}$ is observed. By extrapolation, for distances greater than $10 \mathrm{~mm}$ (where electron density is too low to probe electron properties using the diagnostic) it might be expected that at low discharge current, electron temperatures would remain low $(<0.5 \mathrm{eV})$, while at high discharge current electron temperatures might reach high values $(>2 \mathrm{eV})$.

The separation between the spot and plume regimes has been shown to be dependent on a number of factors, including discharge parameters and anode distance [2, 32, 48]. The 
spot-plume transition may not necessarily be a sharply-defined boundary, as shown by Csiky [2], and the two regimes may be separated by a transition regime, as shown by Kaufman [49]. In characterization of these regimes, we will be guided by the trends in electron properties observed from the ITS measurements and also, discharge metrics. The plume mode is associated with regimes of (i) higher discharge current/flow rate ratios, and (ii) higher voltage oscillations. Recently, Mikellides et al investigated transitions between these regimes in the OrCa2D code on the basis of similar metrics [29].

The observation of the highest electron temperatures at some distance from the orifice is compatible with the plume mode for $8 \mathrm{~A}$ and $16 \mathrm{~A}$ discharge currents. The observation of a monotonically decreasing electron temperature profile with a maximum near the orifice is compatible with the spot mode.

The plume mode has been associated with a sheath extending well beyond the cathode orifice, while the spot mode is characterized by a contracted sheath primarily within the orifice. Although investigations within the orifice are not accessible in the experimental study, the rate of decrease of the electron density beyond the orifice as a function of axial distance (clearly slower for $16 \mathrm{~A}$ than at, for example, $2 \mathrm{~A}$ ) further suggests that the higher current regime at $16 \mathrm{~A}$ has features of a plume regime.

The nature of the regimes may be further investigated by a consideration of the discharge voltage standard deviation. In this work, no measured voltage oscillations exceed $2 \%$ of the mean voltage; this was the threshold described in reference [32] for a spot-plume transition. For all the discharge conditions explored experimentally, the discharge appears to remain in the spot mode, according to this criterion. However, other features (such as the trend in the experimentally-determined electron temperature and density of figure 7 at $8 \mathrm{~A}$ and $16 \mathrm{~A}$ ) may develop before the discharge fully transitions from the spot to the plume mode at higher currents.

An increase in electron density is observed at higher discharge currents. As electron current is the main discharge current contribution inside a cathode discharge, the electron current density is expected to follow the total discharge current trend. Because the electron current density is proportional to both the electron density and the drift velocity, its increase is expected to be correlated with the increase of at least one of these quantities. The results presented show that the increase in discharge current is at least due to an increase in electron density. The electron velocity component along the probed radial direction was too low to be resolved and the component along the cathode axis could not be probed. With these experimental constraints, no comments can be made regarding the contribution of electron drift velocity to the electron current density.

\subsection{Electron properties from $2 D$ simulations}

As mentioned earlier, in these simulations, a variation of the $\mathrm{LaB}_{6}$ pellet temperature is studied. This temperature variation corresponds to a variation of the emission current density $[4,12]$ (see equation (15)).
4.2.1. Emitter temperature exploration. The 2D fluid model was used to simulate the discharge at identical discharge conditions to those explored by ITS. Because the temperature of the $\mathrm{LaB}_{6}$ emissive pellet is a key parameter for the simulation, and one whose measurement with high precision is challenging [44], particular attention was paid to its influence on the electron density and temperature values outside the cathode. The lowest temperature of the pellet was chosen close to values previously measured in our team with a thermocouple. In the simulation, the temperature was varied by $5 \mathrm{~K}$ steps over a temperature range of $100 \mathrm{~K}$ (where possible). Discharge currents from 2 to $16 \mathrm{~A}$ with a xenon flow rate of $0.8 \mathrm{mg} \mathrm{s}^{-1}$ were simulated.

For each set of discharge parameters simulated, the simulation was run for $5 \mathrm{~ms}$, and convergence was verified. Electron temperature and density along the cathode centerline were extracted from the 2D maps of electron properties calculated by the simulation. Snapshots of these maps are provided as examples in Fig 5, for two values of the electron emitter temperature. The axial profiles extracted to perform a parametric study were obtained from a temporal average over the last 1 $\mathrm{ms}$ of the simulation and from a spatial average over the radial length which corresponded to that probed in scattering experiments (up to $1.2 \mathrm{~mm}$ from the cathode axis).

The axial profiles for a selected set of emissive pellet temperatures are shown in figures 8, 9 and 10 for a discharge current of, respectively, $2 \mathrm{~A}, 4 \mathrm{~A}$ and $8 \mathrm{~A}$. The set of emissive pellet temperatures displayed was chosen to maximize readability on the figures. With a 16 A discharge current, the range of tested temperatures was reduced to $50 \mathrm{~K}$ because the discharge systematically turned off above $2060 \mathrm{~K}$. The corresponding results are not presented in this paper.

Some general trends apply to the discharge currents shown in figures 8 to 10 . T he e lectron d ensity a lways decreases monotonically with increased distance from the cathode orifice. When the temperature of the emissive pellet is increased, electron density outside the cathode orifice progressively decreases before reaching a steady low-density profile at high emissive pellet temperature.

The trend in electron temperature changes as the emissive pellet temperature rises. For the presented results, electron temperature decreases with distance from the orifice at low emissive pellet temperature. However, at higher emissive pellet temperatures, the electron temperature first increases at distances close to the cathode orifice before approaching a plateau (clearly visible for the cases at $4 \mathrm{~A}$ and $8 \mathrm{~A}$ ). The position at which this plateau starts to develop approaches the orifice a s e mitter temperature increases. As suggested with the simulation at $2 \mathrm{~A}$ discharge current, it seems that the electron temperature profile returns to a decreasing trend once the plateau has reached the orifice. For the $8 \mathrm{~A}$ discharge condition, the transition from a low $T_{e}$, high $n_{e}$ regime at low emissive pellet temperature to a high $T_{e}$, low $n_{e}$ regime at pellet temperature above $1925 \mathrm{~K}$ may correspond to a transition of the discharge behavior from a spot mode to a plume mode, if we consider electron density and temperature values as the criteria for judging such a mode transition. As shown in figure 5 , the region where a large e lectron temperature is 


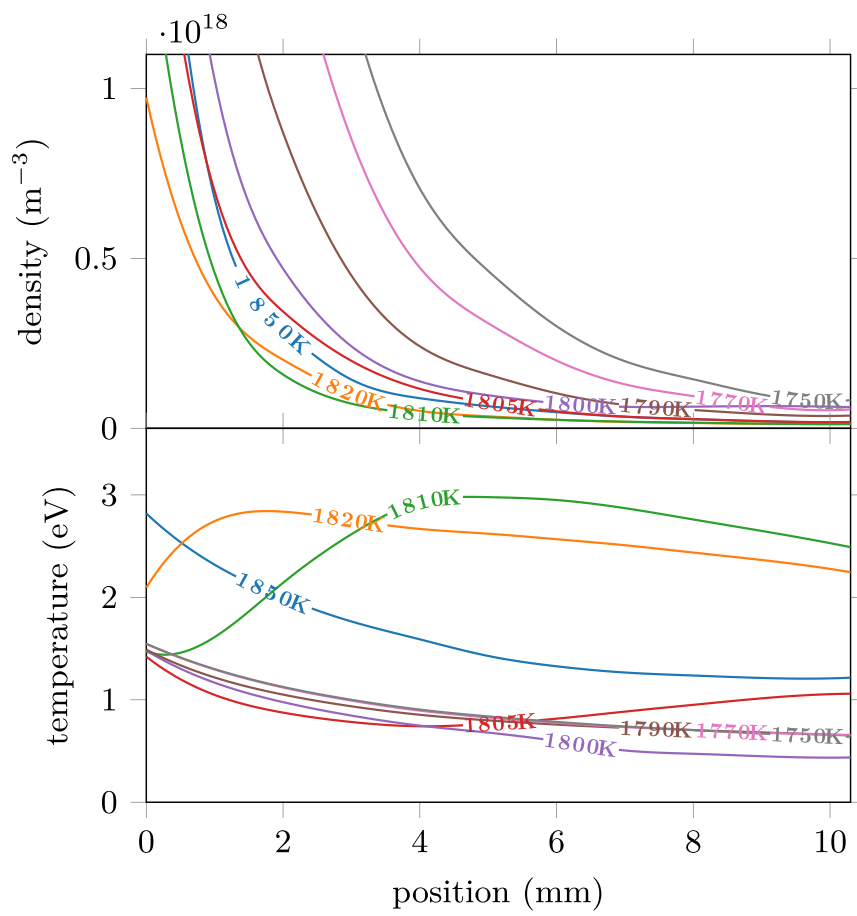

Figure 8. Electron density and temperature obtained with a $2 \mathrm{~A}$ discharge current and a $0.8 \mathrm{mg} \mathrm{s}^{-1}$ xenon flow rate for an emissive pellet temperature varying from $1750 \mathrm{~K}$ to $1850 \mathrm{~K}$.

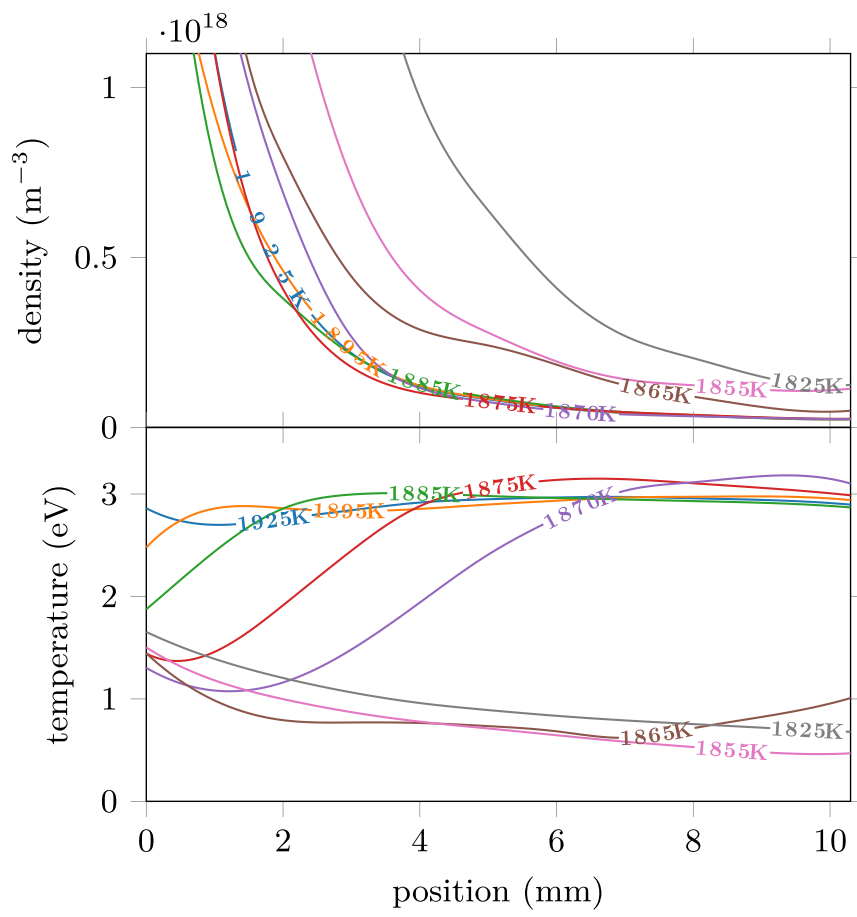

Figure 9. Electron density and temperature obtained with a $4 \mathrm{~A}$ discharge current and a $0.8 \mathrm{mg} \mathrm{s}^{-1}$ xenon flow rate for an emissive pellet temperature varying from $1825 \mathrm{~K}$ to $1925 \mathrm{~K}$.

predicted fills most of the simulated domain outside the cathode orifice. These large values of electron temperature would probably translate into a luminous large plume outside the

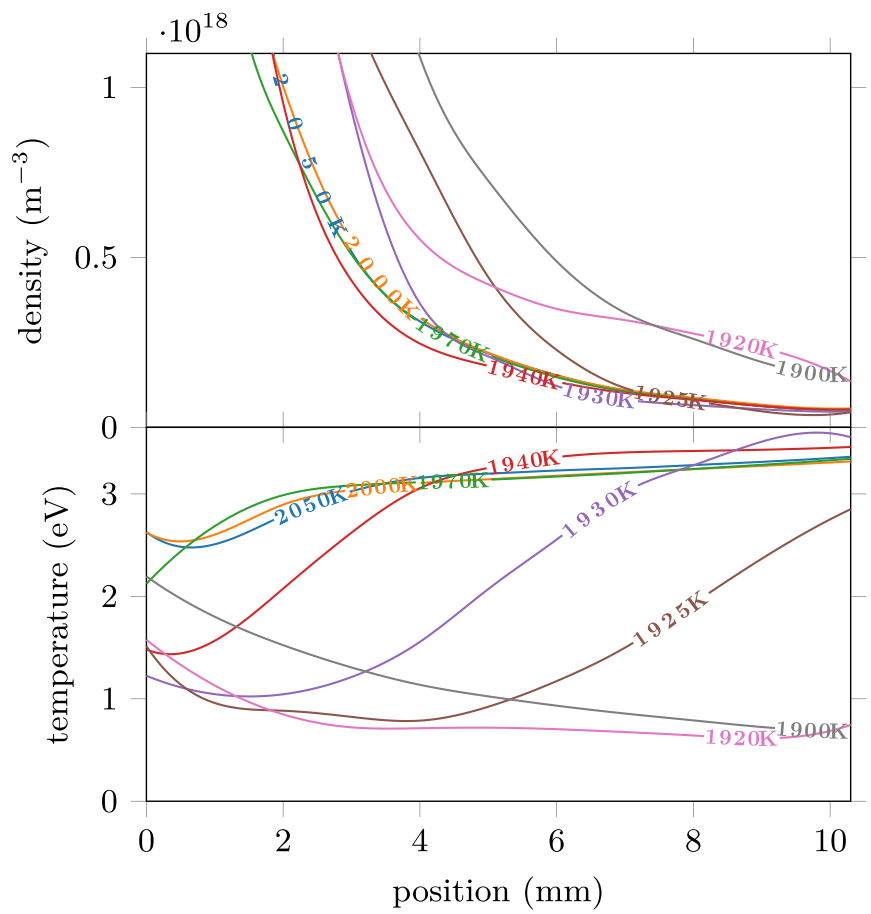

Figure 10. Electron density and temperature obtained with an $8 \mathrm{~A}$ discharge current and a $0.8 \mathrm{mg} \mathrm{s}^{-1}$ xenon flow rate for an emissive pellet temperature varying from $1900 \mathrm{~K}$ to $2000 \mathrm{~K}$.

cathode orifice, commonly observed with a cathode in plume mode.

In the simulation, the discharge current is fixed a nd the varying discharge voltage $U_{d}$ may be examined for information on the different regimes explored. The discharge voltages obtained in the simulation at $8 \mathrm{~A}$ for different emissive pellet temperatures $(1900 \mathrm{~K}, 1925 \mathrm{~K}, 2000 \mathrm{~K}$ and $2050 \mathrm{~K})$ are considered.

The values of mean discharge voltage and standard deviation of these temperatures are, respectively, 44.4(3) V, 43.9(4) V, 39.4(1) V and 39.1(18) V. In experiments, a mean and standard deviation of the discharge voltage of 37.4(5) $\mathrm{V}$ were measured at $8 \mathrm{~A}$ in self-heating mode; the simulation therefore produces voltage values close to those measured experimentally. We can attempt to classify the discharge mode as either plume or spot using the discharge voltage oscillations, as done earlier using the discharge current (where a plume mode was associated with a standard deviation above $2 \%$ ). The increase in pellet temperature from $2000 \mathrm{~K}$ to 2050 $\mathrm{K}$ coincides with a jump in the standard deviation from $0.3 \%$ to $4.7 \%$. This suggests a possible spot-plume mode transition occurring above $2050 \mathrm{~K}$. This transition seems to appear at a different emissive pellet temperature than that previously predicted from the $n_{e}$ and $T_{e}$ profiles (i.e. a pellet temperature of $1925 \mathrm{~K}$ in figure 10 ).

The sensitivity of the discharge mode to the emissive pellet temperature is evident in the simulations. Factors contributing to the thermal balance (the power applied to the heating circuit and the heat shield design) are clearly key elements in the design of a cathode for 
operation in the spot mode, for a specific discharge current range.

4.2.2. Comparison with ITS measurements. The axial profile of electron properties from simulations can first be compared to ITS experimental results for the electron properties obtained for the same discharge current and xenon flow rate. Electron properties derived from the simulation at several emitter temperatures were considered for comparisons.

For a xenon flow rate of $0.8 \mathrm{mg} \mathrm{s}^{-1}$ and discharge currents of $2 \mathrm{~A}, 4 \mathrm{~A}$ and $8 \mathrm{~A}$, the emissive pellet temperatures leading to the best agreement between the electron properties obtained from ITS measurements (shown in figure 7) and simulation result (shown in figures 8 to 10) are, respectively, $1800 \mathrm{~K}, 1860$ $\mathrm{K}$ and $1925 \mathrm{~K}$.

The emitter temperatures leading to the best agreement in electron properties (for the ITS-simulation comparisons) do not correspond to the temperatures that give best agreement in discharge voltage (for ITS-simulation comparisons). In other words, not all the discharge regime features apparent in experiments are captured by the simulations, even though certain behaviors (such as spot-plume transitions) appear to be seen in the simulation. Refinement of the simulation (by selfconsistent coupling of the MIREA plasma and thermal models) will be needed in the future.

Consistently, for the discharge parameters used, the best agreement between simulations and experiments was observed near the transition from a high $n_{e}$, low $T_{e}$ axial profile to a low $n_{e}$, high $T_{e}$ axial profile. The experimental measurements align with a cathode on the verge of a spot-plume transition. Nevertheless, as seen in the previous section, the demarcation between these regimes is not easy to identify using only the amplitude of the discharge voltage oscillations. A high value of $T_{e}$ seems to be observable with a low discharge voltage oscillation level, particularly near the mode transition.

\subsection{Electron properties from Langmuir probe measurements}

The comparisons may be extended with the inclusion of electron property measurements from Langmuir probes. For this, electron density and temperature measurements obtained by Langmuir probes at $8 \mathrm{~A}$ discharge current and a xenon flow rate of $8 \mathrm{mg} \mathrm{s}^{-1}$ were compared to ITS and simulation data for the same operating point. Results are shown in figure 11 . For these discharge parameters, the discharge voltage oscillations were low (below $2 \%$ of the mean discharge voltage) and the discharge was considered to be in the spot mode. The constant nature of the discharge properties was verified with ITS before and after the Langmuir probe measurements.

4.3.1. Comparison with ITS measurements. With measurement uncertainties taken into account, figure 11 shows that the electron density obtained from the Langmuir probe and ITS measurements are in agreement and exhibit the same trend. Nevertheless, this is not the case for the electron temperature, where temperatures measured with the Langmuir probe are four times the values obtained from ITS measurements.
The discrepancy between the ITS and Langmuir probe temperatures may be due to a number of factors.

One important reason might be the change in the discharge mode when the probe was inserted and polarized inside the plasma. When the probe was inserted and kept inside the discharge, large discharge voltage oscillations were observed. These oscillations, with amplitudes as large as $5 \mathrm{~V}$, were observed when the probe was placed at distances $10 \mathrm{~mm}$ and closer to the orifice. At distances $5 \mathrm{~mm}$ and below, these perturbations remained present for up to a minute after extraction of the probe from the plasma. This significant perturbation strongly suggests that probe insertion and measurement were associated with a change of the cathode regime from the spot to the plume mode. This would explain the higher electron temperatures measured with the probe, on the order of those expected in a plume mode [2]. Nevertheless, plume modes are usually associated with lower electron density. The relatively high electron density measured with the probe, despite a possible transition to the plume regime (where lower electron density is expected), might be attributable to the sputtering of the Langmuir probe, leading to a local increase of plasma density.

Another possible explanation for the discrepancy between the ITS and Langmuir probe temperatures could be the directional sensitivity of the ITS measurements (along with the probed wave vector), which is lacking with the Langmuir probe measurements. Due to the axial electric field and low collisionality of the plasma outside the cathode orifice, a nonisotropic electron temperature can be expected with a higher electron temperature along the electric field direction. The electron temperature obtained from ITS would correspond to the low electron temperature along the radial direction, while the values obtained from the Langmuir probe would correspond to an average over all the directions. An ITS measurement with an axial probed wave vector is needed to settle this question. This measurement was not performed because the available viewing ports of the vacuum chamber did not permit such a scattering configuration.

Finally, as mentioned in section 2.3, the estimation of electron temperature with Langmuir probes implies the assumption of a Maxwell-Boltzmann EVDF. Deviation of the true EVDF from this theoretical distribution may contribute to the discrepancy observed. Although no deviation from the Gaussian shape was observed in Thomson scattering spectra, the contribution from a highly energetic electron population (above $3 \mathrm{eV}$ ) might exist, but would not be clearly visible in these Thomson spectra, due to (i) the short spectral range examined for these investigations, in order to facilitate accurate resolution of low electron temperatures, and (ii) the necessity for the highly-energetic electrons to be present at sufficiently high densities for ITS detection.

4.3.2. Comparison with fluids imulations. Results from the Langmuir probe and ITS measurements are also compared to results from simulations in figure 11. Two axial profiles from the simulation have been chosen for the comparison. The first profile, obtained with an emissive pellet temperature of $1925 \mathrm{~K}$, 


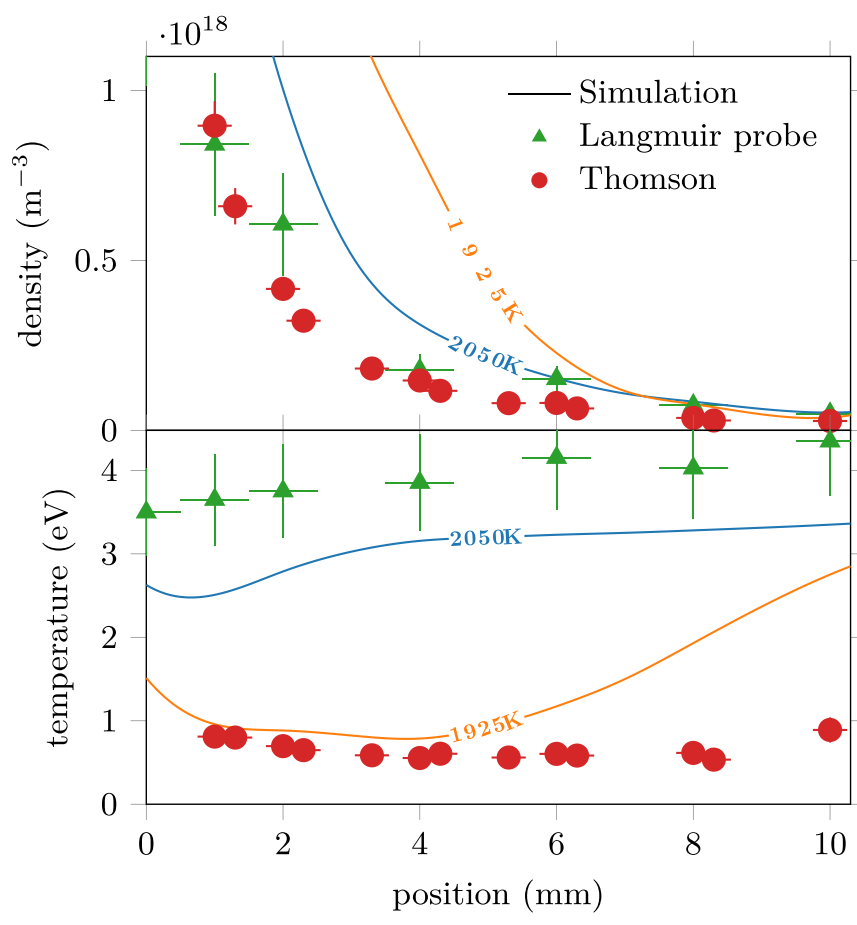

Figure 11. Comparison between electron properties obtained from (i) the fluid simulation at steady state (at two different emitter temperatures, in orange and blue solid lines), (ii) incoherent Thomson scattering experiments, in red filled circles, and (iii) Langmuir probe measurements, in green filled triangles, for a discharge current of $8 \mathrm{~A}$ and flow rate of $0.8 \mathrm{mg} \mathrm{s}^{-1}$.

corresponds to the temperature leading to the best agreement between simulations and ITS measurements, as briefly mentioned in the last section. The second profile, obtained with an emissive pellet temperature of $2050 \mathrm{~K}$, corresponds to a temperature leading to a plume mode of the discharge, with large voltage oscillations. As before, the simulation profiles of electron properties are obtained at steady state $(\approx 10 \mathrm{~ms}$ into the simulation).

Figure 11 shows that there is a good agreement between the axial profiles of $n_{e}$ and $T_{e}$ obtained by Langmuir probe measurements and from simulations when the emissive pellet temperature is fixed at $2050 \mathrm{~K}$. The discharge mode obtained with a $2050 \mathrm{~K}$ emissive pellet temperature is clearly in the plume mode and seems to better simulate the electron properties obtained from Langmuir probe measurements. This observation seems to validate the explanation advanced in the last section for the discrepancy between Langmuir probe and ITS measurements: insertion of the probe inside the plasma switches the discharge from the spot mode to the plume mode. In contrast, the electron temperature obtained from ITS measurements is better simulated with a discharge in a spot mode, with an emissive pellet temperature of $1925 \mathrm{~K}$.

This comparison illustrates once more the sensitivity of agreement to the emitter temperature, which is fixed in the simulation. While the agreement (the order of magnitude of the values obtained in simulation, compared to those experimentally measured) is quite good, further refinements t o t he $\mathrm{c}$ ode $\mathrm{f}$ or $\mathrm{t}$ hermal m odeling c ould likely improve the match between the simulated and measured properties.

\section{Conclusions}

In this work, numerical and experimental tools have been applied to the study of electron properties and regime transitions of a 5 A MIREA $\mathrm{LaB}_{6}$ cathode.

A recently-developed $2 \mathrm{D}$ fluid code has been used to simulate the cathode discharge for various discharge currents and emissive pellet temperatures. These results were used to identify the modification of the discharge behavior during the transition from the spot mode to a plume mode. The emissive pellet temperature has been taken as a parameter allowing a scan of varying emission current densities and a means of exploring the physics associated with different regimes.

The recently-developed THETIS incoherent Thomson scattering diagnostic has been used to measure electron properties of this cathode along the cathode centerline for various discharge currents and xenon flow rates. This marks the first time such a diagnostic has been applied for characterizations of this discharge. At higher discharge currents, electron properties suggesting a transition to a plume mode (a non-monotonic spatial variation in temperature, and a slower decrease in electron density) are observed.

Comparisons between the code and reliable ITS measurements show agreements in general trends, however, improvements to the code will be necessary in the future. The selfconsistent coupling of the thermal model for this cathode to the plasma code (as was achieved for a different cathode architecture [31]) will be important for quantitative comparisons.

Complementary Langmuir probe measurements were performed at one discharge condition used for the ITS and numerical investigations. Here, while the ITS and probe measurements were found to yield similar densities, Langmuir probes gave higher electron temperatures. This discrepancy was attributed to a suspected mode transition induced by the invasive nature of the probe, an effect clearly seen from an increase in discharge voltage oscillations during, and even after, the probe residence time in the plasma. This hypothesis was strengthened by comparison with results from simulations, which suggest that the electron property profiles obtained with the Langmuir probe are more consistent with plume mode features. For an apparently simple, unmagnetized plasma, such as the plume of a cathode, invasive diagnostics may have some shortcomings due to plasma perturbation, rendering their use for code validation risky. The successful application of ITS in this work and its comparison to simulations offers a reliable path forward for advancing cathode modeling.

\section{Acknowledgment}

The authors acknowledge support from CNES and C Boniface and thank K Hara (Stanford University) for discussions. 


\section{ORCID iDs}

Benjamin Vincent (D) https://orcid.org/0000-0001-5420-6002 Sedina Tsikata (D) https://orcid.org/0000-0001-5104-0676 George-Cristian Potrivitu (D) https://orcid.org/0000-00022849-9662

Laurent Garrigues (D) https://orcid.org/0000-0001-6855098X

Stéphane Mazouffre (D) https://orcid.org/0000-0002-82815128

\section{References}

[1] Csiky G A 1969 AIAA 7th Electric Conf., Williamsburg, Virginia, March 3-5 69-258

[2] Csiky G A 1970 J. Spacecraft 7474

[3] Philip C M 1971 AIAA J. 92191

[4] Goebel D M, Watkins R M and Jameson K K 2007 J. Prop. Power 23552

[5] Mikellides I G, Goebel D M, Jorns B A, Polk J E and Guerrero P 2015 IEEE Trans. Plasma Science 43173

[6] Nürmberger F, Hock A and Tajmar M 2015 51st AIAA/SAE/ASEE Joint Conf., July 27-29, 2015, Orlando, Florida 2015-3822

[7] Lev D, Alon G and Appel L 2019 Rev. Sci. Instrum. 90113303

[8] Pedrini D, Misuri T, Tommaso F and Andrenucci M 2017 Aerospace 426

[9] Choueiri E Y 2001 Phys. Plasmas 81411

[10] Mikellides I G, Katz I, Hofer R R and Goebel D M 2013 Appl. Phys. Lett. 102023509

[11] Mikellides I G, Katz I, Goebel D M, Jameson K K and Polk J E 2008 J. Propul. Power 24866

[12] Lafferty J M 1951 J. Appl. Phys. 22299

[13] Jorns B A, Mikellides I and Goebel D 2014 Phys. Rev. E 90063106

[14] Lopez-Ortega A and Mikellides I G 2016 Phys. Plasmas 23043515

[15] Kagan Y M and Perel' V I 1964 Soviet Phys. Uspekhi 6767

[16] Mott-Smith H M and Langmuir I 1926 Phys. Rev. 28727

[17] Fearn D G and Philip C M 1973 AIAA J. 11131

[18] Siegfried D E and Wilbur P J 1978 13th Int. Electric Conf., San Diego, California, 25-27 Apri 1978 78-705

[19] Krishnan M, Jahn R G, von Jaskowsky W F and Clark K E 1977 AIAA J. 151217

[20] Grimaud L, Pétin A, Vaudolon J and Mazouffre S 2016 Rev. Sci. Instrum. 87043506

[21] Carbone E A D, Hübner S, Palomares J M and van der Mullen J J A M 2012 J. Phys. D: Appl. Phys. 45345203

[22] Shneider M N 2017 Phys. Plasmas 24100701

[23] Parks D E, Mandell M J and Katz I 1982 J. Spacecraft 19354

[24] Williams J D and Wilbur P J 1992 J. Spacecraft 29820
[25] Salhi A and Turchi P 1992 28th Joint Conf. and Exhibit Reston, Virigina: American Institute of Aeronautics and Astronautics

[26] Mikellides I G and Katz I 2008 J. Propul. Power 24855

[27] Mikellides I G, Goebel D M, Jorns B A, Polk J E and Guerrero P 2015 IEEE Trans. Plasma Science 43173

[28] Mikellides I, Lopez Ortega A, Goebel D M and Becatti G 2020 Plasma Sources Sci. Technol. 29035003

[29] Mikellides I G, Guerrero P, Ortega A L, Goebel D M and Polk J E 2018 54th AIAA/SAE/ASEE Joint Conf., July 9-11, 2018, Cincinnati, Ohio AIAA-2018-4722

[30] Sary G, Garrigues L and Boeuf J P 2017 Plasma Sources Sci. Technol. 26055007

[31] Sary G, Garrigues L and Boeuf J P 2017 Plasma Sources Sci. Technol. 26055008

[32] Potrivitu G C, Joussot R and Mazouffre S 2018 Vacuum 151122

[33] Pawlik E V and Rawlin V K 1968 J. Spacecraft Rockets 5814

[34] Vincent B, Tsikata S, Mazouffre S, Minea T and Fils J 2018 Plasma Sources Sci. Technol. 27055002

[35] Druyvesteyn M J 1930 Z. Phys. 64 781-98

[36] Magnus F and Gudmundsson J T 2008 Rev. Sci. Instrum. 79073503

[37] Penney C M, Peters R L S and Lapp M 1974 J. Opt. Soc. Am 64712

[38] Herman D A and Gallimore A D 2005 41st AIAA/ASME/SAE/ASEE Joint Conf. \& Exhibit, 10-13 July, Tucson, Arizona, USA AIAA 2005-4252

[39] Popov T K, Dimitrova M, Ivanova P, Hasan E, Horáček J, Dejarnac R, Stöckel J, Weinzettl V and Kovačič J 2014 Contrib. Plasma Phys. 54267

[40] Reid B M and Gallimore A D 2008 44th AIAA/ASME/SAE/ ASEE Joint Conf. \& Exhibit, July 21-23, Hartford, CT, USA AIAA-2008-4920

[41] Lieberman M A and Lichtenberg A J 2005 Principles of Plasma Discharges and Materials Processing, 2nd ed. (New York: Wiley and Sons, Inc.)

[42] Lin T P and Eng G 1989 J. Appl. Phys. 653205

[43] Katz I, Mikellides I G, Polk J E, Goebel D M and Hornbeck S E 2007 J. Propul. Power 23522

[44] Guerrero P, Mikellides I G, Monreal C, Polk J E and Meiron D I 2019 36th Int. Electric Conf., Vienna, Austria, September 15- 19 IEPC-2019-A-301

[45] Mikellides I G, Katz I, Goebel D M and Jameson K K 2007 J. Appl. Phys. 101063301

[46] Davidson R C and Krall N A 1977 Nucl. Fusion 171313

[47] Joussot R, Sary G, Grimaud L, Garrigues L, Mazouffre S, Laurent B, Boniface C, Oriol S and Masson F 2017 35th Int. Electric Conf., Atlanta, Georgia, USA, October 8-12 IEPC-2017-486

[48] Hall S J, Gray T G, Yim J T, Choi M, Mooney M M, Sarver-Verhey T R and Kamhawi H 2019 36th Int. Electric Conf., Vienna, Austria, September 15-19 IEPC-2019-299

[49] Kaufman H R 1975 Technology of electron-bombardment ion thrusters Adv. Electron. Electron Phys. 36 265-373 